\title{
The construction of the coordinated development model of civil aviation industry based on large scale system cybernetics
}

\author{
Zhang $\mathrm{Xu}^{1, \mathrm{a}}$, Du Qing ${ }^{1, \mathrm{~b}}$, Xiong Jing ${ }^{1, \mathrm{c}}$, Zhao Ming ${ }^{1, \mathrm{~d}}$ \\ ${ }^{1}$ Civil Aviation Transportation College, Shanghai University of Engineering Science \\ azx_sues@163.com, ${ }^{\mathrm{b}}$ dqnora@126.com, crystal_420@126.com, ${ }^{d}$ jmmolly_zhao@163.com
}

\begin{abstract}
Keywords: Aviation Industry, Large Scale System Cybernetics, Multi-Variable Coordinated Control Principle, Hierarchical Control Structure.
\end{abstract}

\begin{abstract}
Aviation is an important strategic industry of economic and social development. The issues of imbalances and inconsistence remain outstanding in the current civil aviation development. Other problems restricting the development of aviation industry include unreasonable airspace distribution, slow infrastructure development, insufficient professional storage, enterprise competitive weakness, management to be straightened out. To this end, based on analysis of the bottleneck problems of harmonious development of the civil aviation industry, this paper analyzes the key factors of the coordinated development, innovatively utilizes the multivariable coordinated principle of the large scale system Cybernetics, constructs change granularity model of the coordinated development of civil aviation industry, and effectively solve the complex problems in the social sciences. This paper also gives suggestions for promoting coordinated development of civil aviation, initially forming a safe, convenient, efficient, green, modern system of civil aviation.
\end{abstract}

\section{Introduction}

Civil aviation system is an huge open complex system: firstly, it itself has constant activities to exchange the flow of people, goods and information flow, capital flow with the external environment, reflecting a sharp characteristic of opening. Secondly, civil aviation industry system contains a great number of subsystem, including the civil aviation manufacturing systems, civil aviation and civil aviation transportation system services system. Each system contains a great variety of subsystems, with complex structure. Thirdly, the relationship between subsystems are crossing and overlapping each other. Civil aviation system is a socio-economic system that has not only the typical General characteristics of large-scale systems (large, complex structures, integrated functions, numerous factors, etc.) but also its own characteristics (such as the double restriction of the natural environment and social environment with an obvious openness).

The fundamental cause of these problems is that the developing direction of the civil aviation industry, management methods, and industrial policy is not fully viewed from the perspective of sustainable development, which ignored the systematic feature of the civil aviation industry. Each department is running on an automated track, pursuing the maximization of local interests, rather than optimizing the civil aviation industry system, let alone combining the civil aviation industry with the national economic and social development. All these eventually lead to reciprocal restrictions instead of mutual promotion. The development bottleneck appears constantly.

\section{Large systems cybernetics theory of multi-variable coordinated control}

The hierarchical control structure is commonly adopted in large system coordinated control. As shown in Fig. 1, $J$ is the overall goal (overall task); $R$ is the general constraint (total resources); $\varepsilon_{i}, \quad \varepsilon_{j}$ is deviation error ; $\lambda_{i}, \lambda_{j}$ is coordinated variable; $m_{i}, m_{j}$ is hypothetical association(hypothetical variables); $\sum_{j \neq i}^{N} A_{i j} x_{j} \quad \sum_{i \neq j}^{N} A_{j i} x_{i}$ is actual association. 


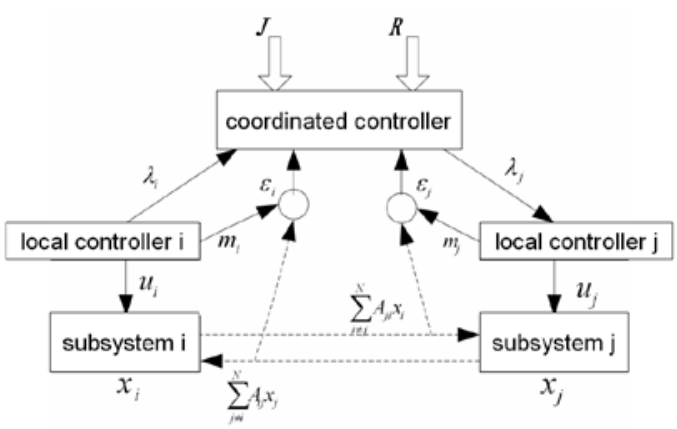

Fig.1 the coordinated control of Large-scale hierarchical systems

$u_{i}, \quad u_{j}$ is local control $(i=1,2, \cdots, N) ; x_{i}, x_{j}$ is local state $(j=1,2, \cdots, N)$. Supposing the large-scale system is made up of $\mathrm{N}$ subsystems, the equitation of state is as follows:

$\dot{x_{i}}=A_{i i} x_{i}+B_{i} u_{i}+\sum_{j \neq i}^{N} A_{i j} x_{j}$

In this formula, $x_{i}, u_{i}$ is the state vector and control vector of the

$i$ th subsystem; $A_{i i}, B_{i}$ are the object matrix and the control matrix of the $i$ th subsystem; $\sum_{j \neq i}^{N} A_{i j} x_{j}$ is the actual association(coupling) of the ${ }^{i}$ th subsystem.

\section{Variable granularity model building of civil aviation coordinated development and control}

The coordinated development of civil aviation industry can reflect basic conditions of coordinated development of the civil aviation industry and its subsystems; provide basic (statistic) relevant indicators for the problems existing in the coordinated development of civil aviation industry, namely the key indicators reflecting the coordinated development of civil aviation industry from perspectives of business, finance, efficiency and quality. The key elements are below analyzed and illustrated on macro, neutral, micro level.

Large System Cybernetics is the combination of system science and computer science, especially the combination of two frontier in each field - the Large Scale System and artificial function, which focus on the common law of various large scale system control process of common law. For this, the large system cybernetics can be used to establish comprehensive evaluation index system of the coordinated development of the modern civil aviation industry, as shown in figure 8. Based on the research of the actual demand of coordinated development and control of modern civil aviation industry, the state space model of various granularity is established, which could be further refined from two perspectives of controlling and being controlled, as shown in Fig. 2. This model could reflect whether the target status is "coordinated", "basically coordinated" or "uncoordinated".

\section{Construction and implementation of the state space model of the coordinated development of} civil aviation industry

\subsection{Construction of multi-layer state space model}

Multi-layer state space model. For the civil aviation industry system, we can adopt hierarchical processing, divide the industry system into different levels of subsystems, with each subsystem establishing the corresponding relationship of input and output according to the index system of hierarchical relationships, so as to build a practical mathematical model of accuracy. The multi-layer state space model of the civil aviation industry system can be built according to the index system shown in table 1.

Each layer of the state space model. 


$$
\left\{\begin{array}{l}
\dot{X}=A X+B U \\
Y=C X+D U
\end{array}\right.
$$

In this formula, $X=\left[x_{1}, x_{2}, x_{3}, \cdots, x_{n}\right]^{T}$ is state variable, $U=\left[u_{1}, u_{2}, u_{3}, \cdots, u_{m}\right]^{T}$ is input control variable, $Y=\left[y_{1}, y_{2}, y_{3}, \cdots, y_{p}\right]$ is output variable, $A, B, C, D$ are respectively coefficient matrix.

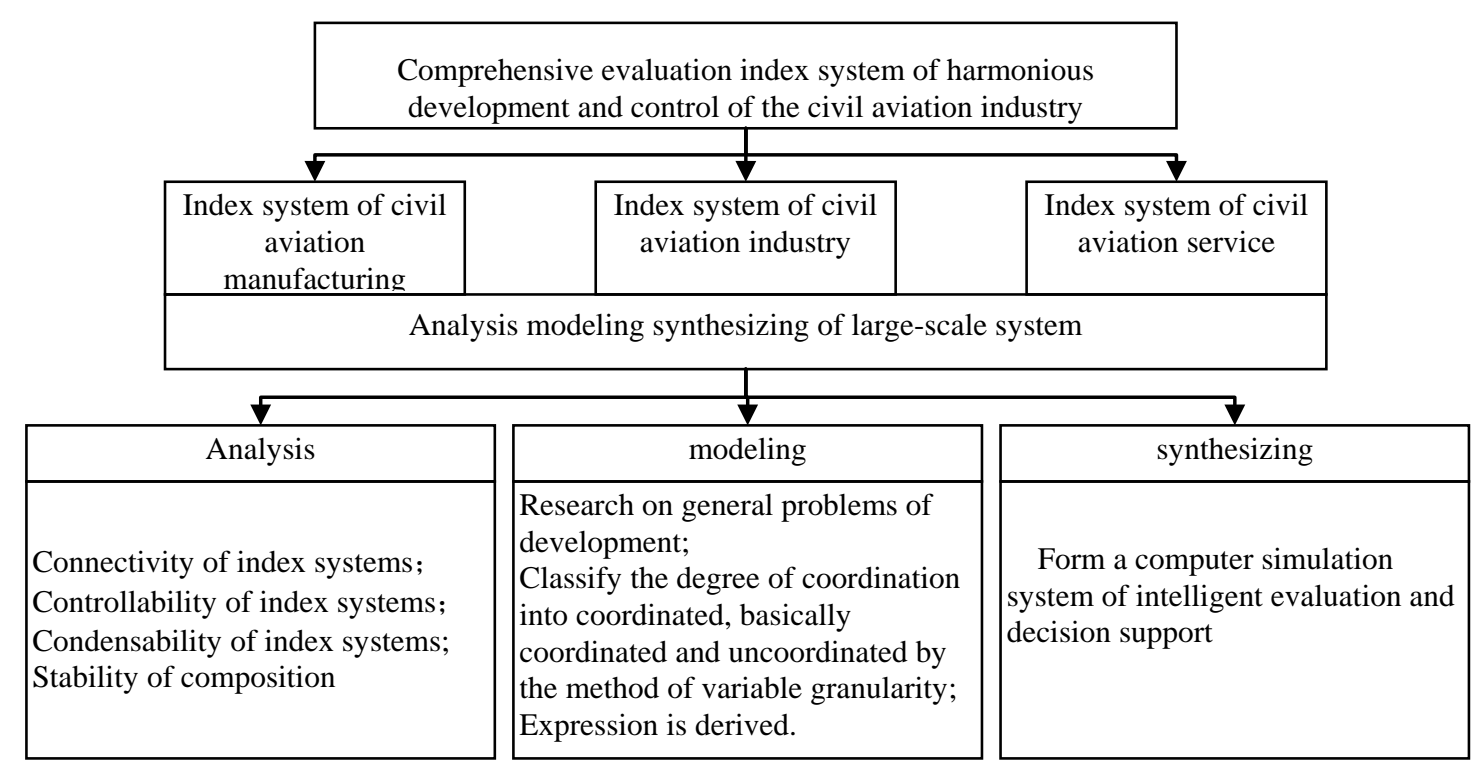

Fig. 2 model of variable granularity of coordinated development and control of civil aviation industry 4.2 The realization of the state space model of the coordinated development of civil aviation industry

Index of the first layer is represented by letter $u$ and subscript $i$ with number; index of the second layer is represented by letter $u$ and subscript $i i$ with number; index of the third layer is represented by letter $u$ and subscript $i i i$ with number; index of the fourth layer is represented by letter $u$ and subscript iv with number; total degree of coordinated development of the civil aviation industry is indicated with $u_{v 1}$. Index system of the coordinated development of the whole civil aviation industry and its code .

Take the same number of state variables as the input of each layer in state space model, with each state variable corresponding with its input variable; State variable should be the relative quantity of the total impact of the outside (except input variable) on the system. In this model, it is the combination of all the influential factors corresponding to input variable. corresponding to input variables $u_{i k}(k=1,2,3, \cdots, 107) 、 u_{i i k}(k=1,2,3, \cdots, 15) 、 u_{i i i k}(k=1,2,3)$ 、 the state variables of $u_{i v 1}$ are $x_{i k}(k=1,2,3, \cdots, 107) 、 x_{i i k}(k=1,2,3, \cdots, 15) 、 x_{i i i k}(k=1,2,3) 、 x_{i v 1}$ respectively。

The actual data of index of the first level of the model $u_{i k}(k=1,2,3, \cdots, 107)$ can be acquired by searching literature. The index of the first level, namely the first layer of input, should be standardized to interval $[0,1]$ through the index of coordinated development according to the actual date. For $u_{i 1}$, based on empirical data and expert knowledge, a data $u_{i 1 \max }$ most in favor of the coordinated development of the system and a data $u_{i 1 \text { min }}$ least in favor of the coordinated development of the system can be determined. Supposing its actual data is $u_{i 1 s j}$, then:

$$
u_{i 1}=\frac{u_{i 1 s j}-u_{i 1 \min }}{u_{i 1 \max }-u_{i 1 \min }}
$$


Likewise, the other indexes of the first level can be standardized in the same way. The main calculated results.Coordination degree falling in interval $[0,0.2]$ indicates poor system coordination; coordination degree in interval $[0.2,0.4]$ indicates a system coordination that is not good; interval $[0.4,0.6]$ indicates normal system coordination; interval $[0.6,0.8]$ in indicates good system coordination and interval $[0.8,1]$ indicates excellent system coordination.

\section{Conclusion}

With the deepening of the reform and development of civil aviation industry and the changing global situation, research and development work is never ended. There are still many problems worth studying and exploring:

(1) Civil aviation belongs to the domain of "the big move", sharing the properties of public utilities, public welfare services, network natural monopoly. Therefore, on the basis of this study, it is worth investigating the coordinated development of public utility and commercial attribute of the civil aviation industry; encouraging the market main body to provide public utilities and public welfare service and ensuring the basic demand of related groups traveling by air.

(2) Seize the opportunity of re-layout of global industry, promote the coordinated development of inland trade, investment, technology innovation. Innovate the mode of processing trade and speed up the formation of a system and mechanism conducive to the development of inland industrial cluster. Support the development of the inland cities in opening international routes and developing multimodal transport, forming a foreign economic corridor running across the Eastern, the Middle and the Western economic region and connecting the north and south part of china. Push forward the customs clearance collaboration between the inland and coastal areas, realizing the information exchange, mutual recognition and enforcement of mutual regulation between related management departments of ports.

(3) Study on the depth development of military and civilian integration in the reform of airspace management system. According to the current airspace management system, the airspace of civil aviation transport is constrained too much by the air force management. It is worth exploring the way to establish unified leadership, demand docking and resource sharing mechanism promoting the development of military and civilian integration, fundamentally solving the problem of flight delay caused by air traffic control, which is mainly due to insufficient airspace available for China's civil aviation. Meanwhile, general aviation development has gradually shows its potential of becoming the new economic growth point in China.

\section{References}

[1] Zhang Xu, Zhao Ming, Kuang Shimin, Du qing. Building a Petri network model of Airline Company Fuel-saving[J]. Advanced Materials Research 2013. Vols. 616-618 ,pp 1107-1110.

[2] Zhang $\mathrm{Xu}$, Zhenling $\mathrm{Xu}$, Ming Zhao. Airline fleet planning model under the target of reducing fuel emissions[J]. Proceeding of International Conference on Social Science and Environmental Protection(SSEP),pp503-506, November 2012.

[3] Xu Zhang, Zhenling Xu, Ming Zhao. Airline fleet planning model under the target of reducing fuel emissions[J]. Proceeding of International Conference on Social Science and Environmental Protection(SSEP),pp503-506, November 2012.

[4] James I. Hileman.Jeremy B. Katz. Payload fuel energy efficiency as a metric for aviation environmental performance [J]. International Congress of the Aeronautical Sciences (2008).

[5] Chen tuansheng. "Analysis of multi-airport operating characteristics and development strategies". China Civil Aviation,2008 vol 7, pp.59-61. 